\title{
Highly permeable monolith-based multichannel in-tip microextraction apparatus for simultaneous field sample preparation of pesticides and heavy metal ions in environmental waters
}

\author{
Lei Chen, Zhuozhuo Wang, Junxian Pei, Xiaojia Huang*
}

State Key Laboratory of Marine Environmental Science, Key Laboratory of the Ministry of Education for Coastal and Wetland Ecosystem, College of the Environment and Ecology, Xiamen University, Xiamen 361005, China

\author{
*Corresponding author. Tel: 086-0592-2189278; Fax: 086-0592-2183137 \\ E-mail: hxj@xmu.edu.cn \\ Corresponding Address: P. O. Box 1009, Xiamen University, Xiamen 361005, China
}

Figure S1. The FT-IR spectrum (a), SEM image (1000×magnification) (b) and SEM image (5000×magnification) (c) of MBS Page S-3

Figure S2. The pore size distribution plot of synthesized MBS

Page S-4

Figure S3. The effect of the sample volume on the extraction performance of MBS/ITMA for pesticides

Page S-5

Figure S4. The effect of the sample volume on the extraction performance of MBS/ITMA for heavy metal ions Page S-6

Figure S5. The effect of the flow rate of adsorption on the extraction performance of MBS/ITMA for pesticides Page S-7

Figure S6. The effect of the flow rate of adsorption on the extraction performance of MBS/ITMA for heavy metal ions

Page S-8

Figure S7. The effect of the eluent on the extraction performance of MBS/ITMA for pesticides Page S-9

Figure S8. The effect of the eluent on the extraction performance of MBS/ITMA for heavy metal ions

Page S-10

Figure S9. The effect of the eluent volume on the extraction performance of MBS/ITMA for pesticides Page S-11 
Figure S10. The effect of the eluent volume on the extraction performance of MBS/ITMA for heavy metal ions

Page S-12

Figure S11. The effect of sample $\mathrm{pH}$ value on the extraction performance of MBS/ITMA for pesticides Page S-13

Figure S12. The effect of sample $\mathrm{pH}$ value on the extraction performance of MBS/ITMA for heavy metal ions Page S-13

Figure S13. The effect of salt concentration on the extraction performance of MBS/ITMA for pesticides Page S-14

Table S1. The chemical properties of studied pesticides Page S-15

Table S2. Temperature programs of GFAAS Page S-17

Table S3. The influences of the preparation parameters on the extraction performance and permeability of MBS Page S-18

Table S4. Comparison of adsorption capacities $(\mu \mathrm{g} / \mathrm{g})$ of MBS and reported adsorbents

Page S-19

Table S5. Comparison of the flow rate $(\mathrm{mL} / \mathrm{min})$ used in the current method and reported approaches

Page S-20

Table S6. Results of determination and recoveries of real water samples spiked with studied pesticides

Page S-21

Table S7. Results of determination and recoveries of real water samples spiked with studied heavy metal ions

Page S-22

Table S8. Comparison of proposed method with reference methods for the analysis of target analytes in water samples

Page S-23

Standards and reagents Page S-24

Instruments Page S-24

Chromatographic conditions and AAS information for the analysis of studied pesticides and metal ions

Page S-25

The measurement of the adsorption capacity of MBS

Page S-25

References

Page S-26 

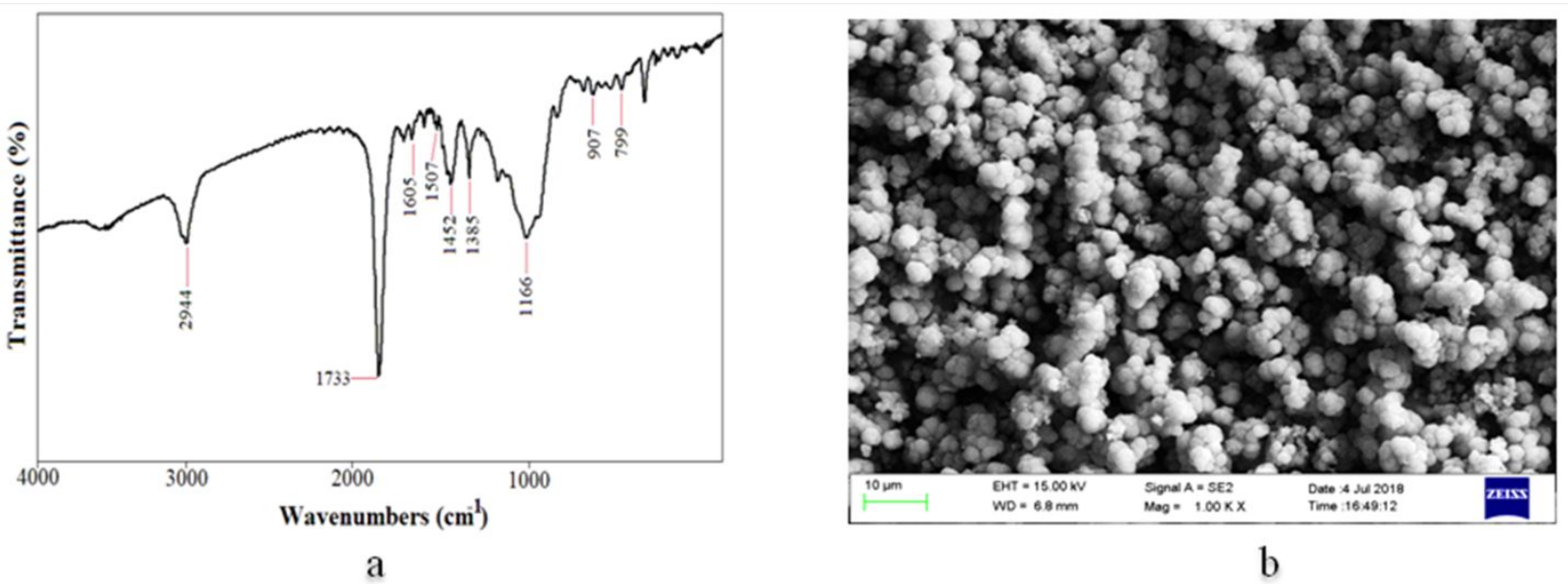

a

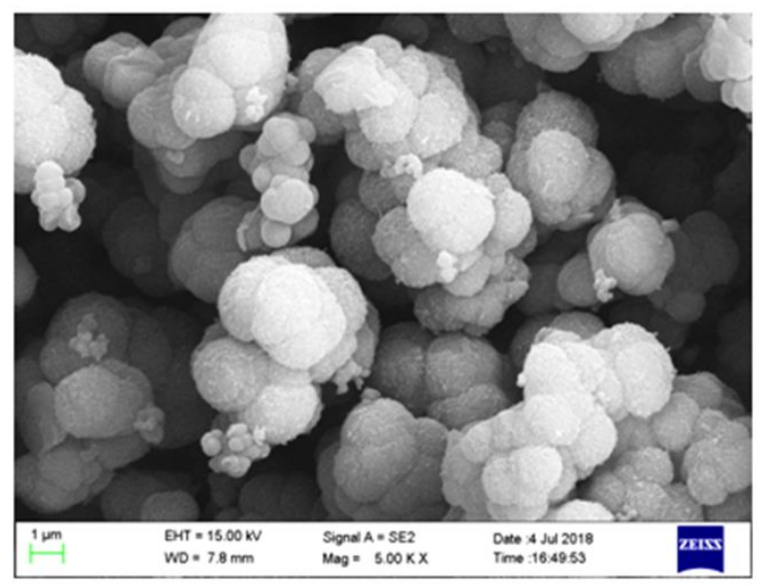

c

Figure S1. The FT-IR spectrum (a), SEM image $(1000 \times$ magnification) $($ b) and SEM image $(5000 \times$ magnification $)$ of MBS $(c)$ 


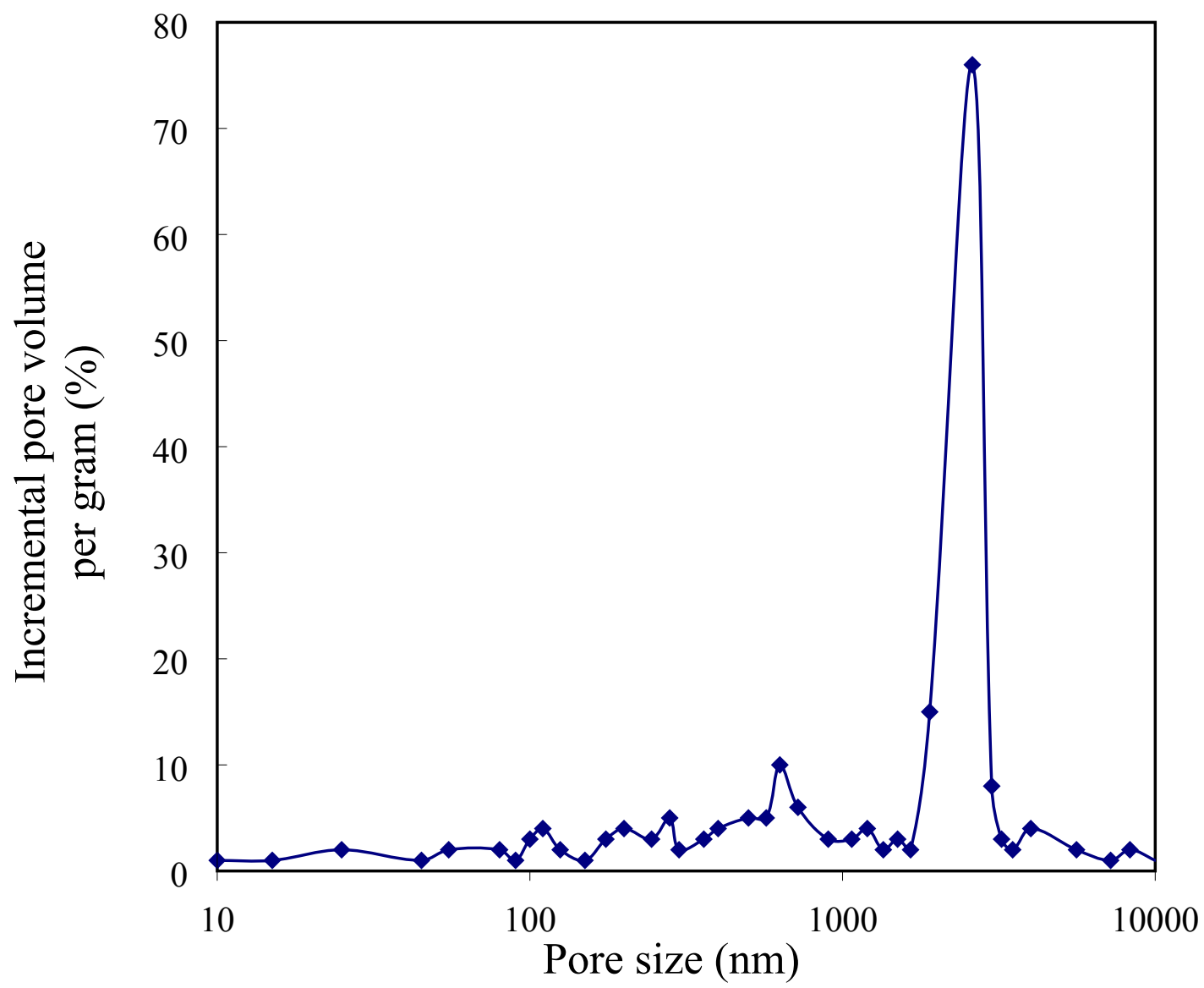

Figure S2. The pore size distribution plot of synthesized MBS 


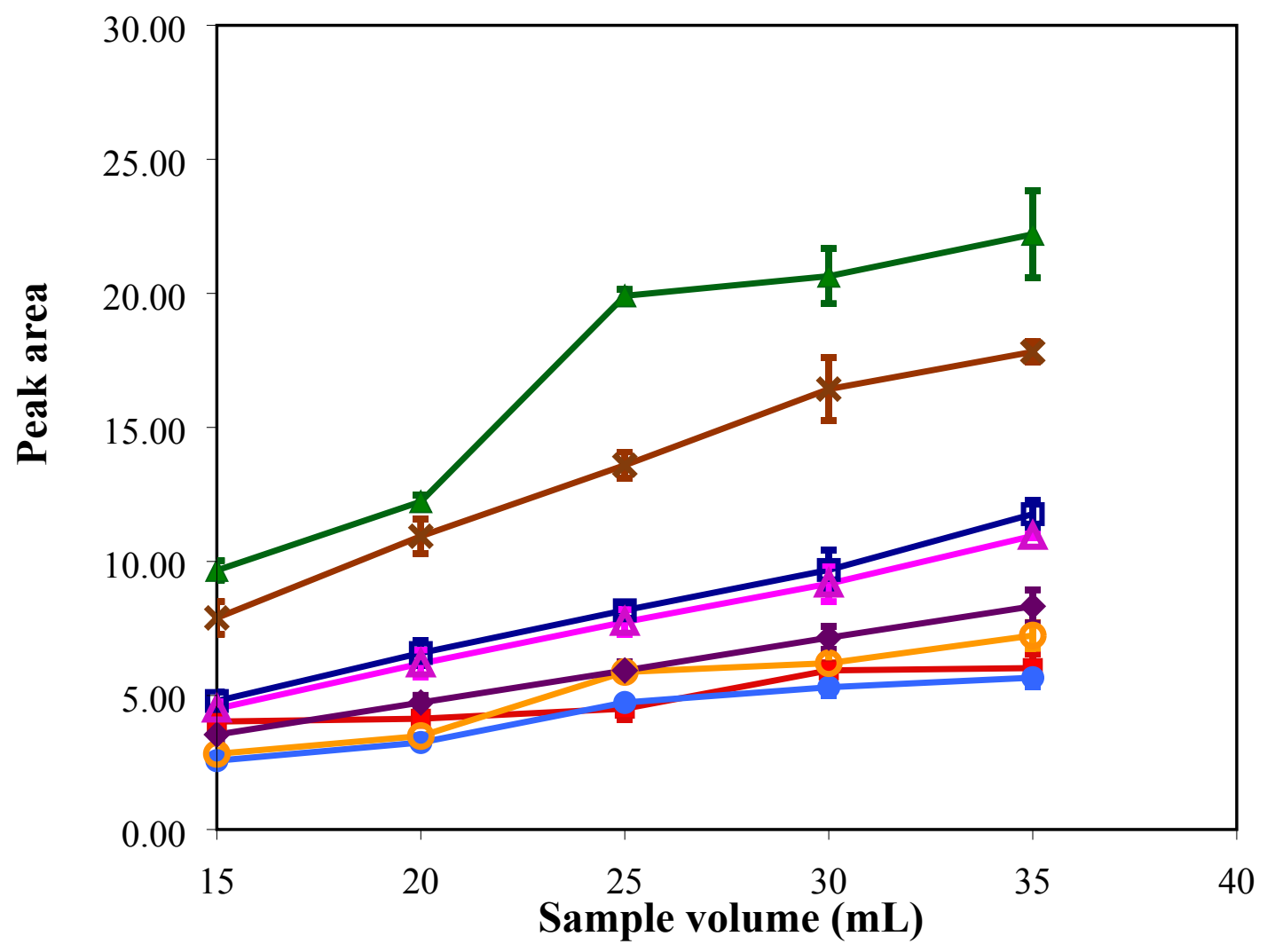

Figure S3. The effect of the sample volume on the extraction performance of MBS/ITMA for pesticides

Conditions: the eluent volume (methanol/FA=99/1.0, v/v) was $100 \mu \mathrm{L}$; the adsorption flow rate was $1.5 \mathrm{~mL} / \mathrm{min}$; sample $\mathrm{pH}$ value did not be adjusted and no salt was added. The spiked concentrations of CAR and the other compounds were 10.0 and $20.0 \mu \mathrm{g} / \mathrm{L}$, respectively.

Symbols: $\square \mathrm{CLO}$; $\triangle \mathrm{CAR}$; $\mathrm{ISO}$; $\bigcirc \mathrm{FEN} ; \diamond \mathrm{TRN}$; $\square \mathrm{MYC} ; \triangle \mathrm{TRF} ; \times$ HEX 


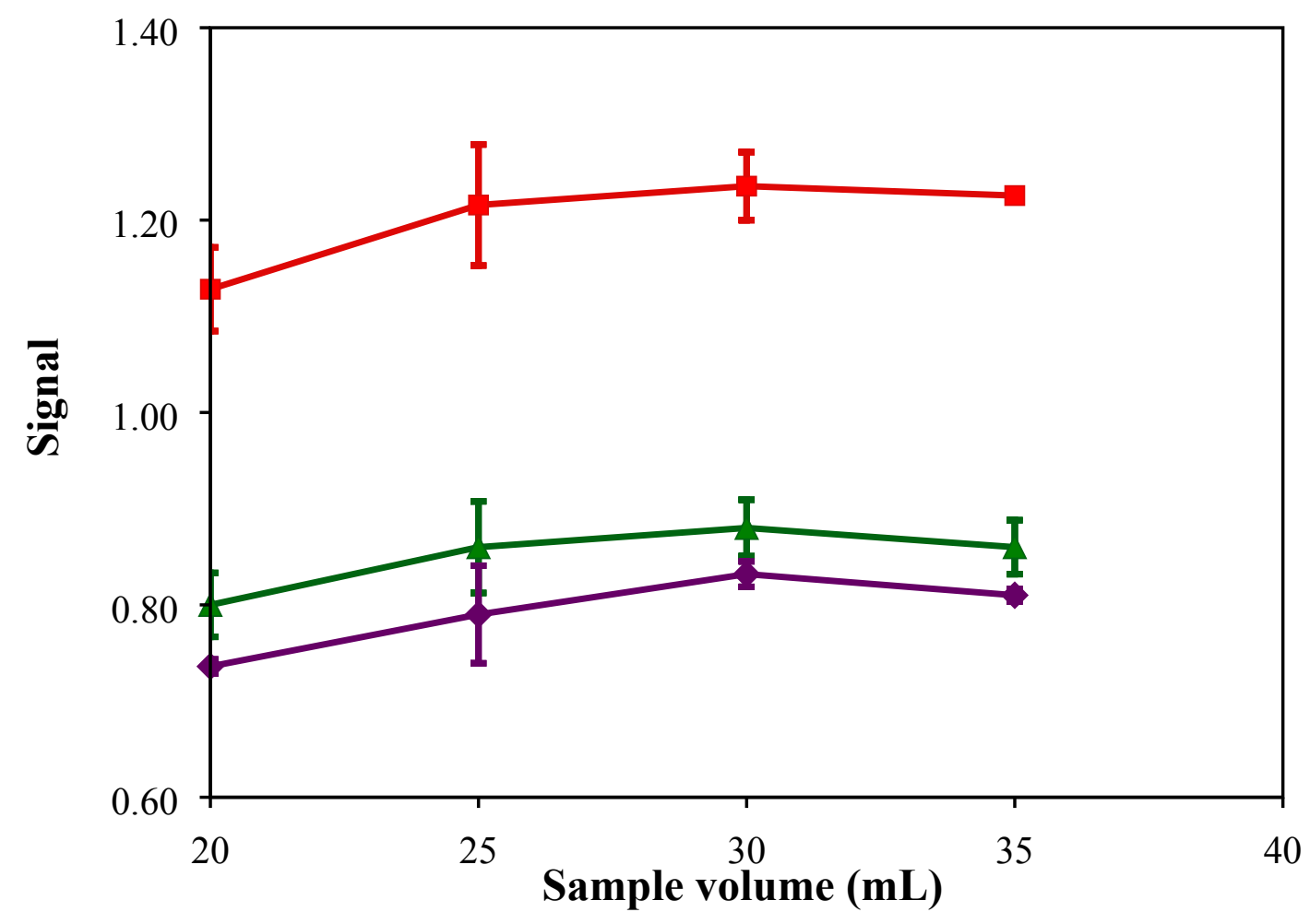

Figure S4. The effect of the sample volume on the extraction performance of MBS/ITMA for heavy metal ions

Conditions: the eluent volume ( $3 \mathrm{~mol} / \mathrm{L} \mathrm{HNO}_{3}$ containing $0.5 \%$ EDTA) was $300 \mu \mathrm{L}$; the adsorption flow rate was $1.5 \mathrm{~mL} / \mathrm{min}$; sample $\mathrm{pH}$ value did not be adjusted. The spiked concentrations of $\mathrm{Cd}(\mathrm{II}), \mathrm{Pb}(\mathrm{II})$ and $\mathrm{Cu}(\mathrm{II})$ were $0.2,0.4$ and $0.2 \mu \mathrm{g} / \mathrm{L}$, respectively.

Symbols: $\square \mathrm{Cd}(\mathrm{II}) ; \boldsymbol{\Delta} \mathrm{Pb}(\mathrm{II}) ; \diamond \mathrm{Cu}(\mathrm{II})$. 


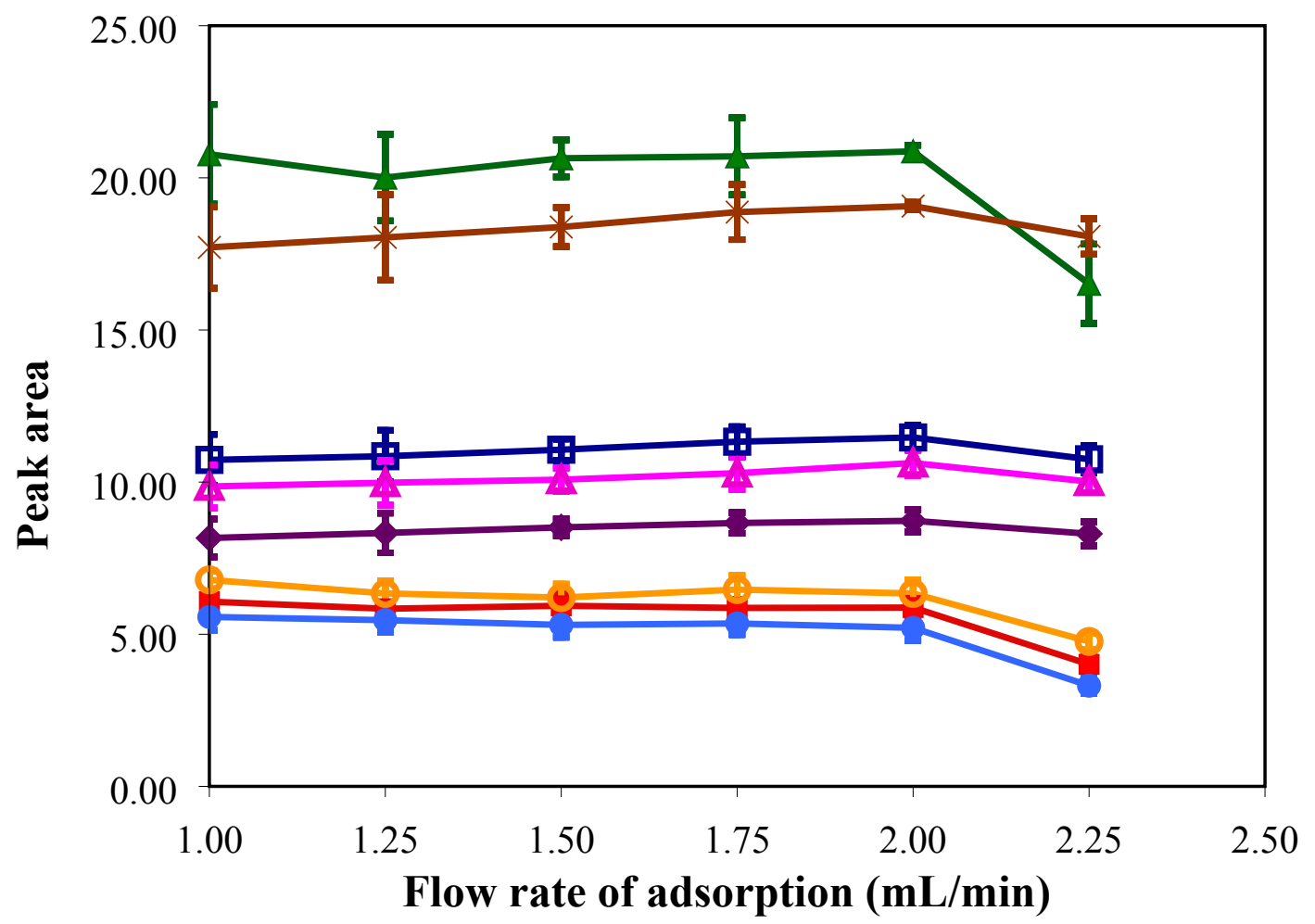

Figure S5. The effect of the flow rate of adsorption on the extraction performance of MBS/ITMA for pesticides

Conditions: the sample volume was $30.0 \mathrm{~mL}$; the other conditions were the same as in Figure S3.

Symbols: $\square \mathrm{CLO} ; \triangle \mathrm{CAR}$ - $\mathrm{ISO} ; \bigcirc \mathrm{FEN} ; \diamond \mathrm{TRN}$; $\square \mathrm{MYC} ; \triangle \mathrm{TRF} ; \times$ HEX 


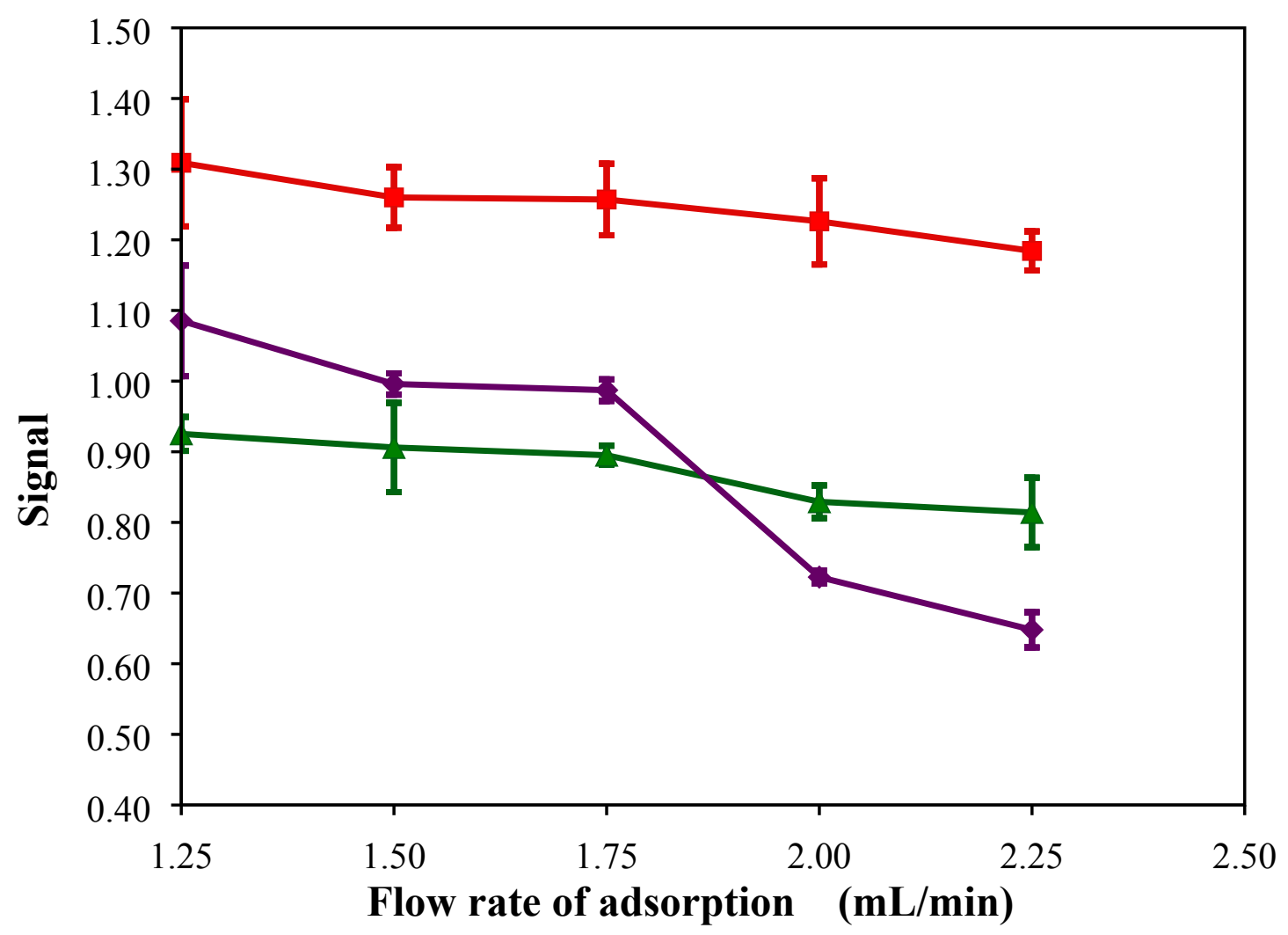

Figure S6. The effect of the flow rate of adsorption on the extraction performance of MBS/ITMA for heavy metal ions

Conditions: the sample volume was $30.0 \mathrm{~mL}$; the other conditions were the same as in Figure S4.

Symbols: $\boldsymbol{C} \mathrm{Cd}(\mathrm{II}) ; \boldsymbol{\Delta} \mathrm{Pb}(\mathrm{II}) ; \diamond \mathrm{Cu}(\mathrm{II})$. 


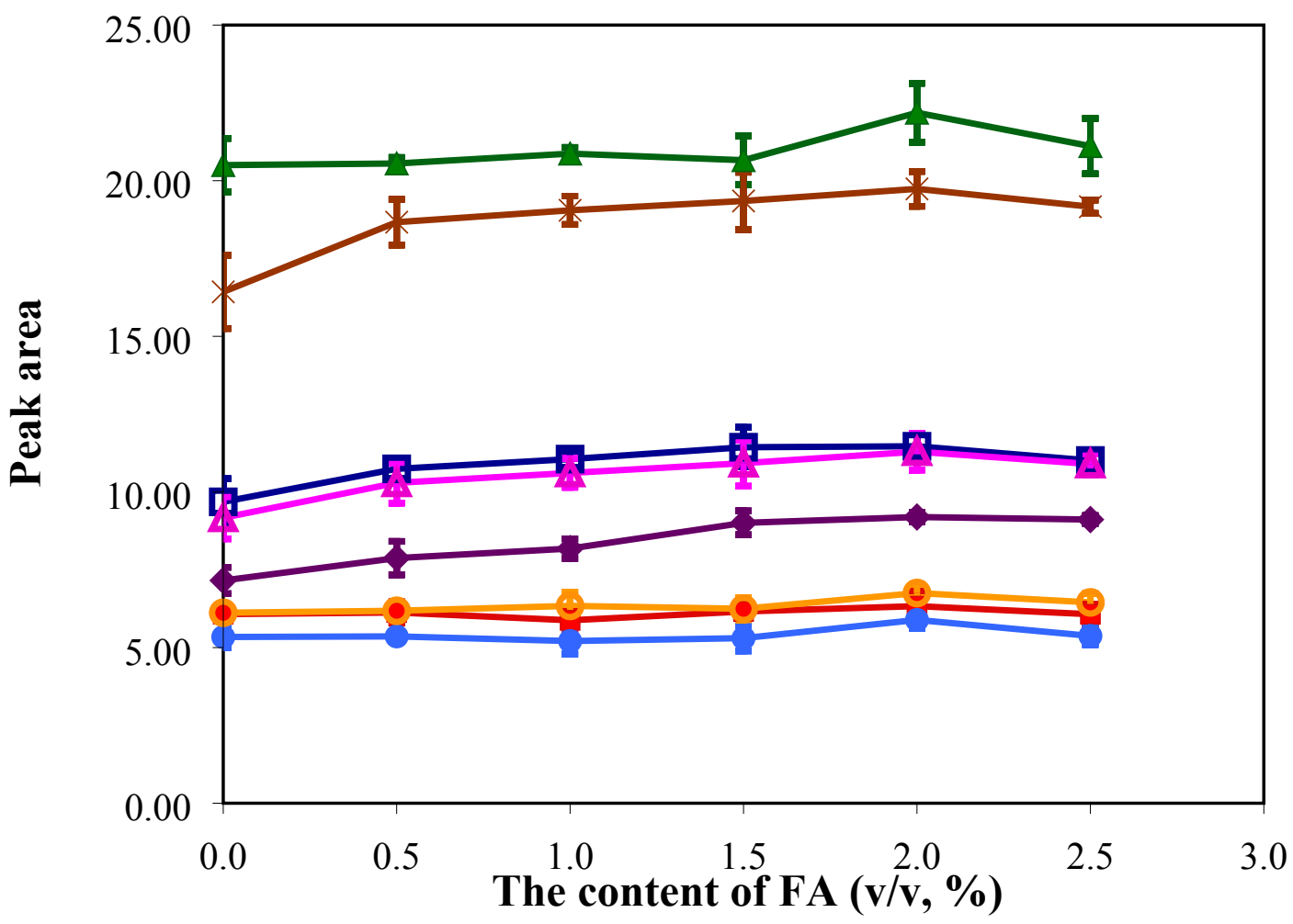

Figure S7. The effect of the eluent on the extraction performance of MBS/ITMA for pesticides

Conditions: the flow rate of adsorption was $2.0 \mathrm{~mL} / \mathrm{min}$; the other conditions were the same as in Figure S5.

Symbols: $\square \mathrm{CLO}$; $\triangle \mathrm{CAR}$; $\bullet \mathrm{ISO}$; $\bigcirc \mathrm{FEN} ; \diamond \mathrm{TRN}$; $\square \mathrm{MYC} ; \triangle \mathrm{TRF} ; \times$ HEX 


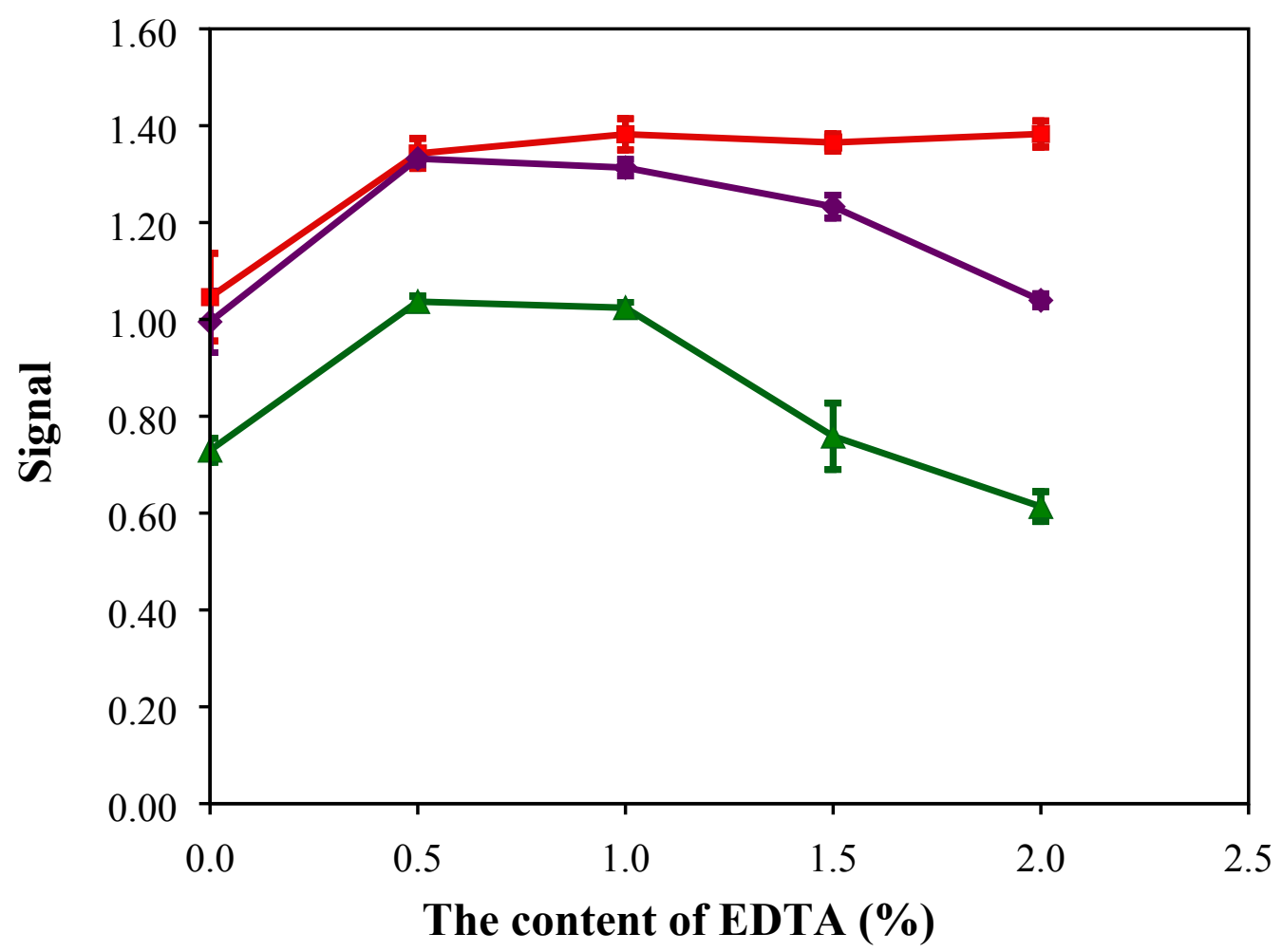

Figure S8. The effect of the eluent on the extraction performance of MBS/ITMA for heavy metal ions

Conditions: the flow rate of adsorption was $1.75 \mathrm{~mL} / \mathrm{min}$; the other conditions were the same as in Figure S6.

Symbols: $\square \mathrm{Cd}(\mathrm{II}) ; \Delta \mathrm{Pb}(\mathrm{II}) ; \diamond \mathrm{Cu}(\mathrm{II})$. 


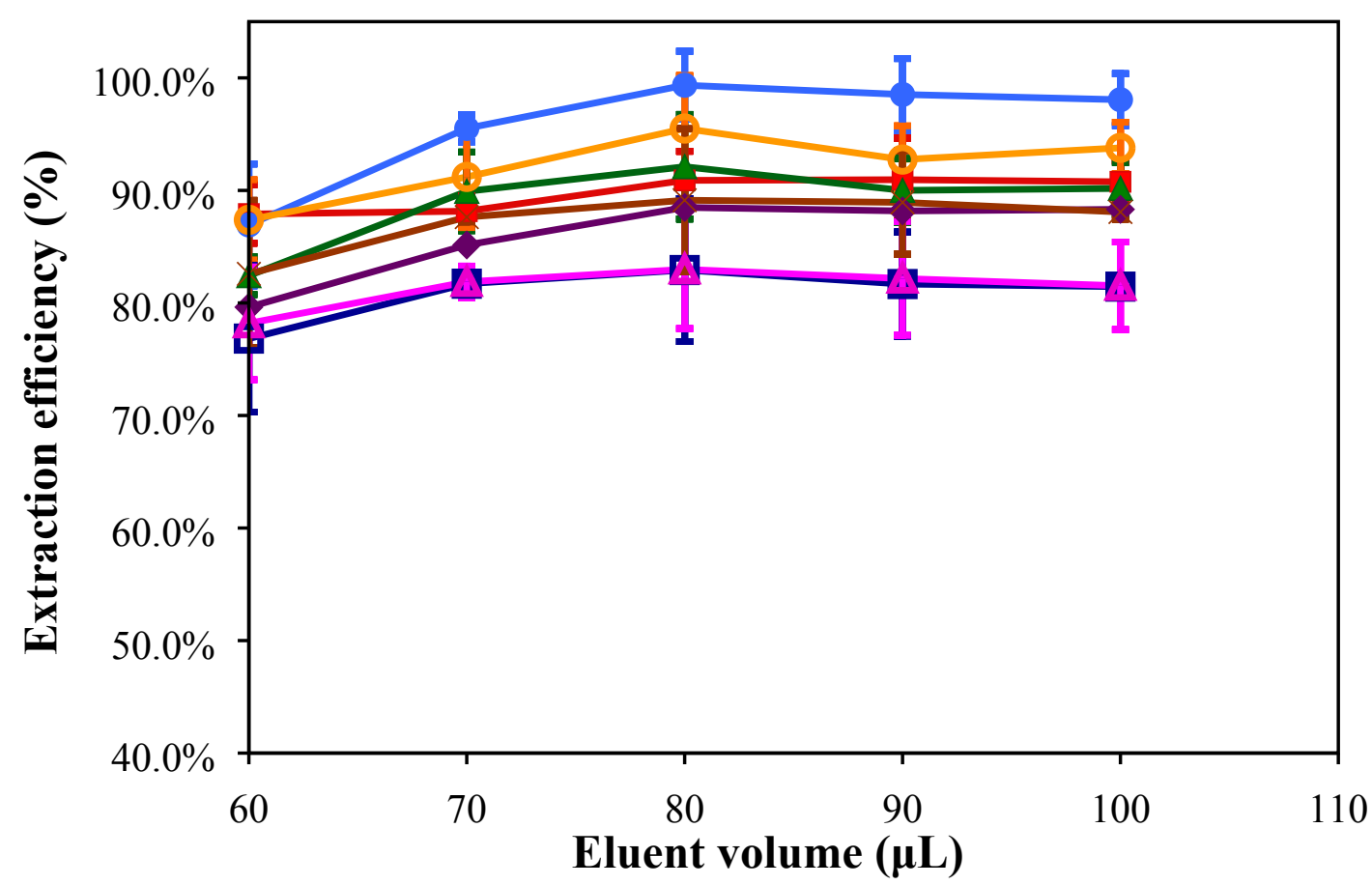

Figure S9. The effect of the eluent volume on the extraction performance of MBS/ITMA for pesticides

Conditions: the eluent was the mixture of methanol/FA=98/2.0 (v/v); the other conditions were the same as in Figure S7.

Symbols: $\square \mathrm{CLO}$; $\triangle \mathrm{CAR}$ - ISO; O FEN; $>\mathrm{TRN}$; $\square \mathrm{MYC} ; \triangle \mathrm{TRF} ; \times$ HEX 


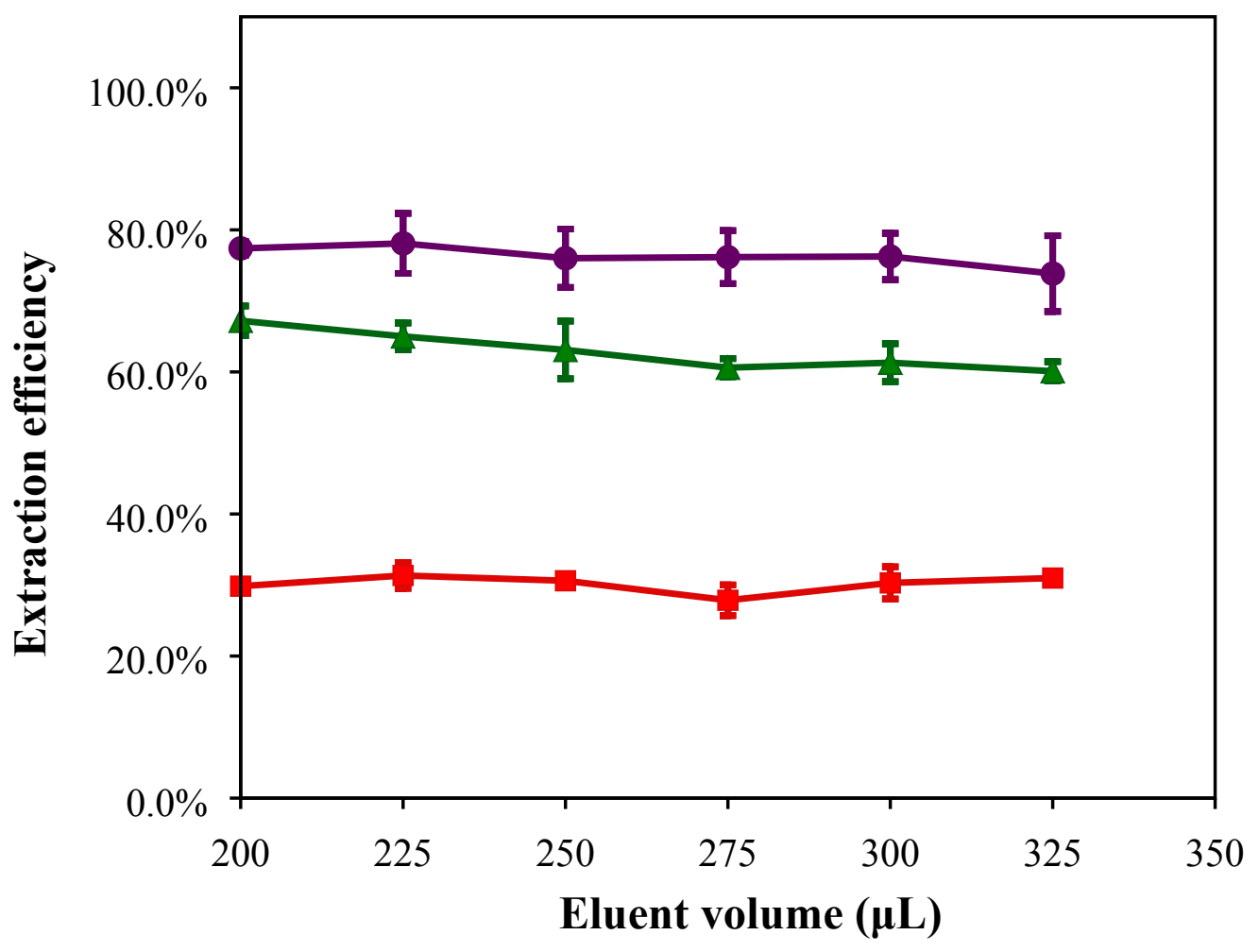

Figure S10. The effect of the eluent volume on the extraction performance of MBS/ITMA for heavy metal ions

Conditions: the eluent was $3 \mathrm{~mol} / \mathrm{L} \mathrm{HNO}_{3}$ containing $0.5 \%(\mathrm{v} / \mathrm{v}) \mathrm{EDTA}$; the other conditions were the same as in Figure S8.

Symbols: $\square \mathrm{Cd}(\mathrm{II}) ; \boldsymbol{\Delta} \mathrm{Pb}(\mathrm{II}) ; \diamond \mathrm{Cu}(\mathrm{II})$. 


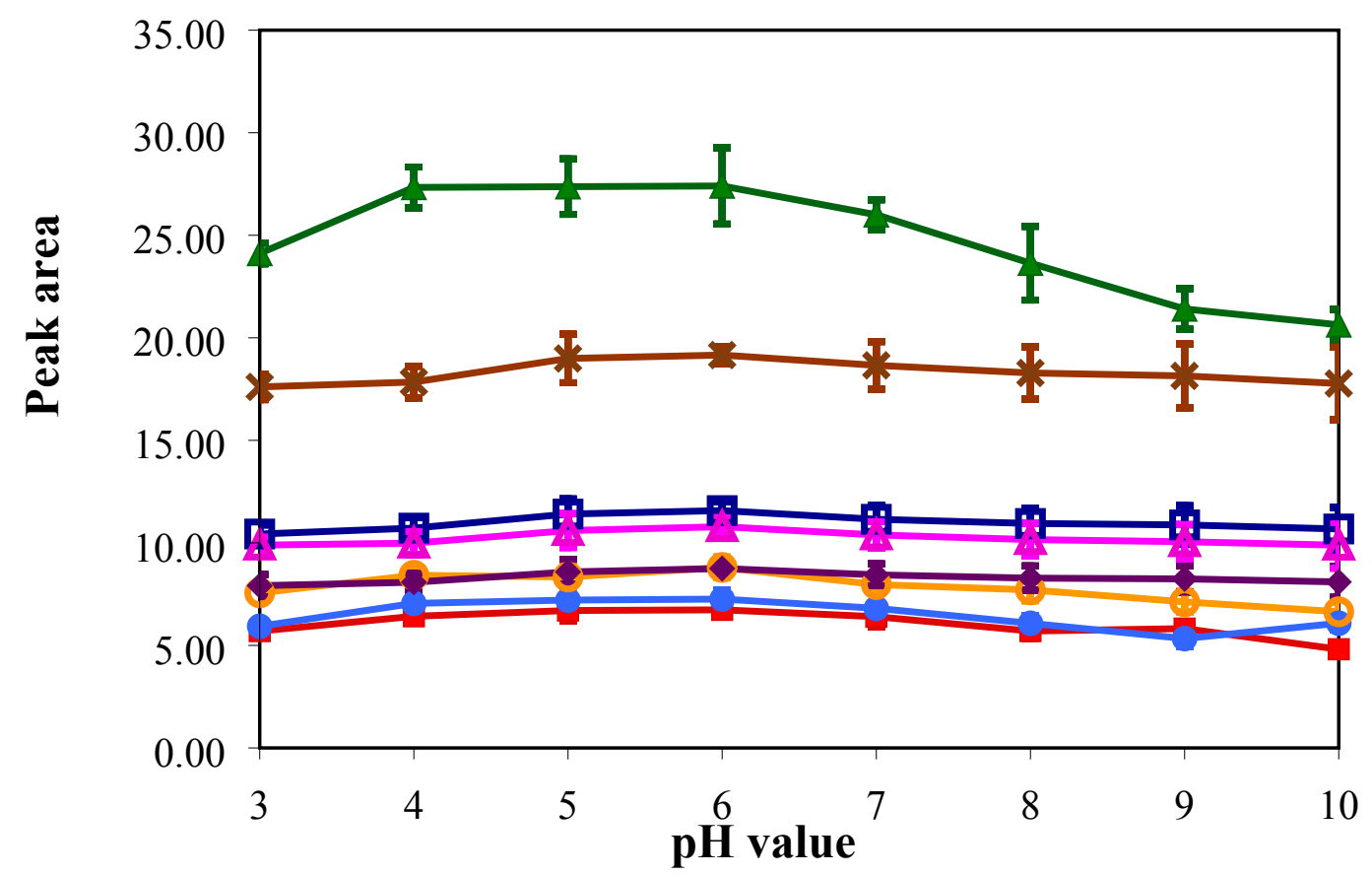

Figure S11. The effect of sample $\mathrm{pH}$ value on the extraction performance of MBS/ITMA for pesticides

Conditions: the eluent was the mixture of methanol/FA=98/2.0 (v/v); the other conditions were the same as in Figure S9.

Symbols: $\square \mathrm{CLO}$; $\triangle \mathrm{CAR}$ ○ ISO; O FEN; $\diamond \mathrm{TRN}$; $\square \mathrm{MYC} ; \triangle \mathrm{TRF} ; \times$ HEX

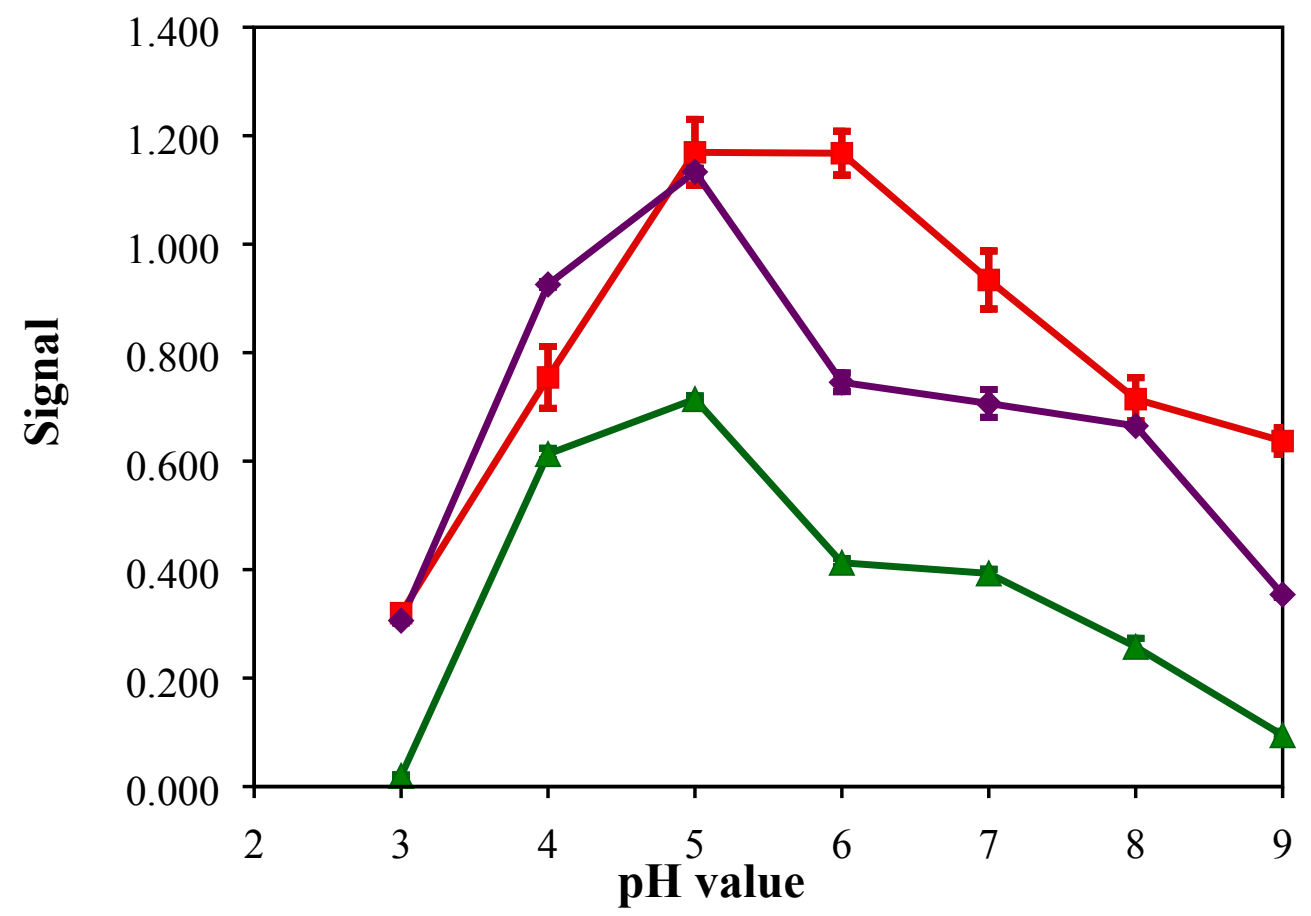

Figure S12. The effect of sample $\mathrm{pH}$ value on the extraction performance of 
MBS/ITMA for heavy metal ions

Conditions: the eluent was $3 \mathrm{~mol} / \mathrm{L} \mathrm{HNO}_{3}$ containing $0.5 \%(\mathrm{v} / \mathrm{v})$ EDTA; the other conditions were the same as in Figure S10.

Symbols: $\boldsymbol{C} \mathrm{Cd}(\mathrm{II}) ; \Delta \mathrm{Pb}(\mathrm{II}) ; \diamond \mathrm{Cu}(\mathrm{II})$.

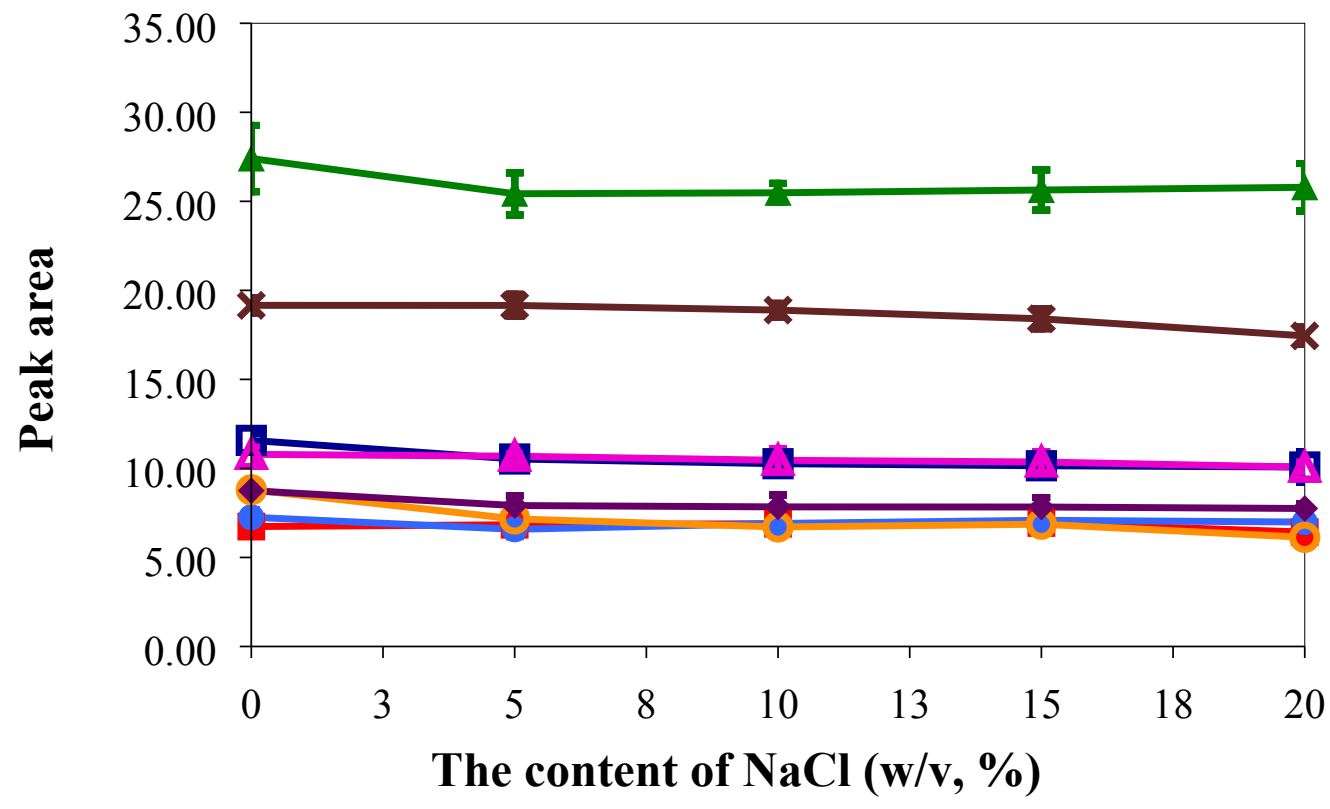

Figure S13. The effect of salt concentration on the extraction performance of MBS/ITMA for pesticides

Conditions: sample $\mathrm{pH}$ value was adjusted to 5.0. The other conditions were the same as in Figure S-5a.

Symbols: $\square \mathrm{CLO} ; \triangle \mathrm{CAR} ; \bullet \mathrm{ISO} ; \bigcirc \mathrm{FEN} ; \diamond \mathrm{TRN} ; \square \mathrm{MYC} ; \triangle \mathrm{TRF} ; \times$ HEX 
Table S1. The chemical properties of studied pesticides

\begin{tabular}{|c|c|c|c|c|c|c|c|}
\hline Compounds & molecular formula & Molecular mass & CAS number & Chemical structures & $\lambda(\mathbf{n m})$ & ${\log K o w^{a}}^{a}$ & $p K a^{a}$ \\
\hline Cloethocarb & $\mathrm{C}_{11} \mathrm{H}_{14} \mathrm{ClNO}_{4}$ & 259.6876 & $51487-69-5$ & & 200 & 1.4 & l \\
\hline Carbaryl & $\mathrm{C}_{12} \mathrm{H}_{11} \mathrm{NO}_{2}$ & 201.22 & $63-25-2$ & & 210 & 2.36 & $12.02^{b}$ \\
\hline Isoprocarb & $\mathrm{C}_{11} \mathrm{H}_{15} \mathrm{NO}_{2}$ & 193.24 & $2631-40-5$ & & 200 & 2.31 & $12.22^{b}$ \\
\hline Fenoxycarb & $\mathrm{C}_{11} \mathrm{H}_{15} \mathrm{NO}_{3}$ & 209.2417 & $72490-01-8$ & & 200 & 4.3 & l \\
\hline Triadimenol & $\mathrm{C}_{14} \mathrm{H}_{18} \mathrm{ClN}_{3} \mathrm{O}_{2}$ & 295.76 & $55219-65-3$ & & 200 & 3.28 & 1.97 \\
\hline Myclobutanil & $\mathrm{C}_{15} \mathrm{H}_{17} \mathrm{ClN}_{4}$ & 288.78 & $88671-89-0$ & & 200 & 3.66 & 2.27 \\
\hline
\end{tabular}




\begin{tabular}{|l|l|l|l|l|l|l|}
\hline Triadimefon & $\mathrm{C}_{14} \mathrm{H}_{16} \mathrm{ClN}_{3} \mathrm{O}_{2}$ & 293.75 & $43121-43-3$ & 200 \\
\hline Hexaconazole & $\mathrm{C}_{14} \mathrm{H}_{17} \mathrm{C}_{12} \mathrm{~N}_{3} \mathrm{O}$ & 314.21 & $79983-71-4$ & 200 \\
\hline
\end{tabular}

\section{a. Calculated via the website: Chemicaliae.com}

b. Santalad A., Zhou L., Shang F.J., et al. Micellar electrokinetic chromatography with amperometric detection and off-line solid-phase extraction for analysis of carbamate insecticides. Journal of Chromatography A, 1217 (2010) 5288-5297. 
Argon was used as the inert gas throughout the measurements. The corresponding working currents were 8,10 , and $5 \mathrm{~mA}$ for $\mathrm{Cd}(\mathrm{II}), \mathrm{Pb}(\mathrm{II})$ and $\mathrm{Cu}(\mathrm{II})$, respectively. Slit width was set at $0.5 \mathrm{~nm}$ for the target ions. GFAAS temperature programs for respective metals were listed in Table $\mathrm{S} 2$.

Table S2. Temperature programs of GFAAS

\begin{tabular}{cccc}
\hline Element [wavelength] & Step & Temperature $/{ }^{\circ} \mathrm{C}$ & Holding $/ \mathrm{s}$ \\
\hline $\mathrm{Cd}(\mathrm{II})[228.8 \mathrm{~nm}]$ & Volatilize & 100 & 30 \\
& Ash & 350 & 20 \\
& Atomize & 1100 & 3 \\
& Clean & 2500 & 3 \\
$\mathrm{~Pb}(\mathrm{II})[217 \mathrm{~nm}]$ & Volatilize & 100 & 30 \\
& Ash & 350 & 20 \\
& Atomize & 1300 & 3 \\
& Clean & 2500 & 3 \\
$\mathrm{Cu}(\mathrm{II})[324.8 \mathrm{~nm}]$ & Volatilize & 100 & 30 \\
& Ash & 400 & 20 \\
& Atomize & 2150 & 3 \\
& Clean & 2500 & 3 \\
\hline
\end{tabular}


Table S3. The influences of the preparation parameters on the extraction performance and permeability of MBS

\begin{tabular}{|c|c|c|c|c|c|c|c|c|c|c|c|c|}
\hline \multirow{2}{*}{ No. } & \multicolumn{2}{|c|}{ Monomers $(\%, \mathrm{w} / \mathrm{w})$} & \multicolumn{2}{|c|}{ Cross-linkers $(\%, \mathrm{w} / \mathrm{w})$} & \multicolumn{4}{|c|}{ Peak area } & \multicolumn{3}{|c|}{ Signal } & \multirow{2}{*}{ Permeability } \\
\hline & AMD & AT & ED & DB & FEN & CAR & MYC & TRF & $\mathrm{Cd}^{2+}$ & $\mathrm{Pb}^{2+}$ & $\mathrm{Cu}^{2+}$ & \\
\hline 1 & 50 & 50 & 0 & 100 & 0.8 & 29.0 & 1.7 & 18.8 & 1 & 1 & 1 & very poor \\
\hline 2 & 50 & 50 & 33 & 67 & 8.2 & 81.2 & 8.2 & 16.4 & I & I & I & poor \\
\hline 3 & 50 & 50 & 50 & 50 & 14.1 & 142 & 16.9 & 21.7 & I & I & 1 & Good \\
\hline 4 & 50 & 50 & 67 & 33 & 14.3 & 131 & 15.8 & 20.5 & I & I & l & poor \\
\hline 5 & 50 & 50 & 100 & 0 & 6.5 & 89.0 & 7.1 & 17.3 & 1 & 1 & I & poor \\
\hline 6 & 0 & 100 & 50 & 50 & 16.3 & 132 & 17.4 & 17.4 & 0.89 & 0.81 & 1.33 & poor \\
\hline 7 & 33 & 67 & 50 & 50 & 13.5 & 123 & 15.0 & 20.2 & 1 & / & 1 & Good \\
\hline 8 & 50 & 50 & 50 & 50 & 18.6 & 146 & 18.6 & 21.3 & I & I & I & Good \\
\hline 9 & 67 & 33 & 50 & 50 & 19.7 & 158 & 20.2 & 22.3 & 1.17 & 1.07 & 1.84 & very good \\
\hline 10 & 100 & 0 & 50 & 50 & 8.3 & 109 & 10.4 & 21.7 & 0.16 & 0.66 & 0.06 & Good \\
\hline
\end{tabular}

Note: the sample matrix was ultrapure water, and the spiked concentrations for each pesticide was $100 \mu \mathrm{g} / \mathrm{L}$, for $\mathrm{Cd}^{2+}, \mathrm{Cu}^{2+}$ and $\mathrm{Pb}^{2+}$ were $0.5,0.5,1.0 \mu \mathrm{g} / \mathrm{L}$, respectively .

The ratio of monomers to cross-linkers was kept at 40/60 (w/w), and the ratio of monomer mixture (including monomers and cross-linkers to porogenic solvent was kept at 30/70 (w/w). 
Table S4. Comparison of adsorption capacities $(\mu \mathrm{g} / \mathrm{g})$ of MBS and reported adsorbents

\begin{tabular}{|c|c|c|c|c|}
\hline Methods & Adsorbents & Compounds & Adsorption capacities $(\mu \mathrm{g} / \mathrm{g})$ & Ref. \\
\hline SPME-HPLC/UV & Acryloyled $\beta$-cyclodextrin hybrid monolith & Carbaryl & 2250 & [1] \\
\hline SPME-HPLC/UV & Acryloyl $\beta$-cyclodextrin monolithic column & Carbaryl & 580 & [2] \\
\hline On-line SPE-HPLC/UV & Zeolite NaY modified with cetyltrimethylammonium bromide & Carbaryl & 1500 & [3] \\
\hline $\mathrm{SPE} / \mathrm{FAAS}$ & Naphthalene methyltrioctyl ammonium chloride & $\mathrm{Cu}^{2+}$ & 100 & {$[4]$} \\
\hline $\mathrm{CME}^{\mathrm{a} / \mathrm{ICP}-\mathrm{MS}}$ & N-(2-aminoethyl)-3-aminopropyltrimethoxysilane-silica & $\mathrm{Cu}^{2+}$ & 114 & {$[5]$} \\
\hline SPE/FAAS & Octadecyl silica & $\mathrm{Cu}^{2+}$ & 51.9 & {$[6]$} \\
\hline $\begin{array}{c}\text { MBS/ITMA-HPLC/DAD } \\
\text { MBS/ITMA-GFAAS }\end{array}$ & Poly (AT/AMD-co-DB/ED) & $\begin{array}{c}\text { Carbaryl } \\
\mathrm{Cu}^{2+}\end{array}$ & $\begin{array}{c}6022 \\
164\end{array}$ & $\begin{array}{l}\text { this } \\
\text { work }\end{array}$ \\
\hline
\end{tabular}

a: capillary microextraction. 
Table S5. Comparison of the flow rate $(\mathrm{mL} / \mathrm{min})$ used in the current method and reported approaches

\begin{tabular}{|c|c|c|c|c|}
\hline Methods & Adsorbents & Compounds & $\begin{array}{l}\text { Flow rate } \\
(\mathrm{mL} / \mathrm{min})\end{array}$ & Ref. \\
\hline PMMEb-HPLC/UV & Poly (butyl methacrylate-co-1,6-hexanediolethoxylatediacrylate) monolith & Phenylurea herbicides & 0.6 & [8] \\
\hline $\mathrm{M}-\mathrm{SP} \mu \mathrm{E}^{\mathrm{c}} / \mathrm{UV}-\mathrm{V}$ is & Activated carbon xerogel & $\mathrm{Hg}$ & 0.3 & [10] \\
\hline $\mathrm{PT}^{\mathrm{d}}$-SPE-HPLC/UV & Sol-gel hybrid adsorbent & Cyanazine, Atrazine & 0.4 & [11] \\
\hline $\begin{array}{c}\text { SPME/ICP-MS } \\
\text { on-line CME/ICP-MS }\end{array}$ & $\begin{array}{l}\text { Carboxyl-functionalized organic-inorganic hybrid monolithic column } \\
\text { Poly (GMA-EDMA-NH2) monolithic capillary }\end{array}$ & $\begin{array}{c}\mathrm{Cr}, \mathrm{Sb} \\
\mathrm{Se}(\mathrm{IV}), \mathrm{Se}(\mathrm{VI})\end{array}$ & $\begin{array}{l}0.2 \\
0.1\end{array}$ & $\begin{array}{l}{[12]} \\
{[13]}\end{array}$ \\
\hline IT-SPME-HPLC/DAD & Poly (4-vinyl pyridineco-ethylene dimethacrylate) monolith & Triazoles & 0.2 & [15] \\
\hline $\begin{array}{l}\text { MBS/ITMA-HPLC/DAD } \\
\text { MBS/ITMA/GFAAS }\end{array}$ & Poly (AT/AMD-co-DB/ED) & $\begin{array}{c}\text { Pesticides } \\
\mathrm{Cd}^{2+}, \mathrm{Pb}^{2+}, \mathrm{Cu}^{2+}\end{array}$ & 1.75 & $\begin{array}{l}\text { this } \\
\text { work }\end{array}$ \\
\hline
\end{tabular}

a: tip microextraction device.

b: polymer monolithic microextraction

c: miniaturized solid phase microextraction

d: pipette-tip solid-phase extraction

e: in-tube solid phase microextraction 
Table S6. Results of determination and recoveries of real water samples spiked with studied pesticides

\begin{tabular}{|c|c|c|c|c|c|c|c|}
\hline \multirow{2}{*}{ Analytes } & \multirow{2}{*}{$\begin{array}{c}\text { Spiked } \\
(\mu \mathrm{g} / \mathrm{L})\end{array}$} & \multicolumn{6}{|c|}{ Detected $(\mu \mathrm{g} / \mathrm{L}) /$ recovery $(\% \mathrm{RSD}, \mathrm{n}=3)$} \\
\hline & & \multicolumn{2}{|c|}{ Wastewater } & \multicolumn{2}{|c|}{ River water } & \multicolumn{2}{|c|}{ Seawater } \\
\hline \multirow{4}{*}{ CLO } & 0.0 & 0.068 & & ND & & ND & \\
\hline & 0.10 & 0.17 & $96.8(3.0)$ & 0.080 & $80.1(7.4)$ & 0.11 & $105(1.7)$ \\
\hline & 1.0 & 0.89 & $82.1(4.1)$ & 0.80 & $80.0(5.9)$ & 0.96 & $96.0(7.1)$ \\
\hline & 20 & 22 & $109(6.1)$ & 20 & $98.3(1.7)$ & 22 & $111(2.0)$ \\
\hline \multirow{4}{*}{ CAR } & 0.0 & 0.062 & & ND & & ND & \\
\hline & 0.050 & 0.12 & $107(5.6)$ & 0.042 & $84.9(7.2)$ & 0.042 & $84.3(6.2)$ \\
\hline & 0.50 & 0.60 & $107(5.5)$ & 0.46 & $92.7(7.5)$ & 0.55 & $109(4.4)$ \\
\hline & 10 & 9.9 & $98.1(3.2)$ & 8.9 & $88.7(5.3)$ & 10 & $100(5.4)$ \\
\hline \multirow{4}{*}{ ISO } & 0.0 & 0.062 & & 0.048 & & 0.15 & \\
\hline & 0.10 & 0.17 & $111(2.7)$ & 0.15 & $101(7.7)$ & 0.25 & $102(4.1)$ \\
\hline & 1.0 & 1.1 & $106(8.2)$ & 0.99 & $94.4(4.6)$ & 1.3 & $111(4.4)$ \\
\hline & 20 & 20 & $98.6(4.2)$ & 18 & $91.1(1.9)$ & 22 & $107(7.0)$ \\
\hline \multirow{4}{*}{ FEN } & 0.0 & ND & & ND & & ND & \\
\hline & 0.10 & 0.10 & $99.5(6.7)$ & 0.11 & $107(6.0)$ & 0.10 & $104(3.3)$ \\
\hline & 1.0 & 1.1 & $109(3.3)$ & 0.98 & $97.6(6.4)$ & 1.1 & $109(4.3)$ \\
\hline & 20 & 20 & $98.2(3.1)$ & 17 & 84.9 (3.9) & 18 & $89.2(2.9)$ \\
\hline \multirow{4}{*}{$\mathrm{TRN}$} & 0.0 & 0.96 & & 0.17 & & 0.15 & \\
\hline & 0.10 & 1.1 & $94.4(4.4)$ & 0.28 & $108(6.0)$ & 0.23 & $82.8(7.7)$ \\
\hline & 1.0 & 1.8 & $83.8(2.5)$ & 1.2 & $102(5.5)$ & 1.1 & $99.5(1.2)$ \\
\hline & 20 & 20 & $94.0(5.1)$ & 17 & $82.8(3.8)$ & 16 & $80.8(3.6)$ \\
\hline \multirow{4}{*}{ MYC } & 0.0 & 0.21 & & ND & & 0.024 & \\
\hline & 0.10 & 0.30 & $89.7(7.3)$ & 0.091 & $84.0(4.1)$ & 0.11 & $87.1(4.2)$ \\
\hline & 1.0 & 1.0 & $80.3(5.8)$ & 0.97 & $96.2(4.4)$ & 0.97 & $94.8(2.2)$ \\
\hline & 20 & 19 & $94.4(6.1)$ & 16 & $80.6(7.9)$ & 16 & $81.6(0.7)$ \\
\hline \multirow{4}{*}{ TRF } & 0.0 & 0.12 & & ND & & ND & \\
\hline & 0.10 & 0.23 & $109(5.1)$ & 0.10 & $103(3.9)$ & 0.083 & $83.4(4.1)$ \\
\hline & 1.0 & 0.93 & $80.6(5.2)$ & 0.81 & $80.7(3.0)$ & 0.81 & $80.7(6.3)$ \\
\hline & 20 & 18 & $91.1(7.8)$ & 17 & $86.8(4.1)$ & 16 & $82.0(4.3)$ \\
\hline \multirow{4}{*}{ HEX } & 0.0 & ND & & ND & & ND & \\
\hline & 0.10 & 0.10 & $99.7(2.4)$ & 0.11 & $108(7.7)$ & 0.11 & $112(7.8)$ \\
\hline & 1.0 & 0.90 & $90.2(7.9)$ & 0.88 & $88.0(3.1)$ & 0.82 & $82.1(6.6)$ \\
\hline & 20 & 18 & $87.5(6.2)$ & 17 & $85.3(3.9)$ & 16 & $80.3(4.3)$ \\
\hline
\end{tabular}


Table S7. Results of determination and recoveries of real water samples spiked with studied heavy metal ions

\begin{tabular}{|c|c|c|c|c|c|c|c|}
\hline \multirow{2}{*}{ Samples } & \multirow{2}{*}{$\begin{array}{c}\text { Spiked } \\
(\mathrm{ng} / \mathrm{L})\end{array}$} & \multicolumn{6}{|c|}{ Detected $(\mathrm{ng} / \mathrm{L}) /$ recovery $(\% \mathrm{RSD}, \mathrm{n}=3)$} \\
\hline & & \multicolumn{2}{|c|}{$\mathrm{Cd}^{2+}$} & \multicolumn{2}{|c|}{$\mathrm{Pb}^{2+}$} & \multicolumn{2}{|c|}{$\mathrm{Cu}^{2+}$} \\
\hline \multirow[t]{4}{*}{ Waste water } & 0.00 & 49.0 & & 36.0 & & 52.0 & \\
\hline & 2.50 & 51.4 & $97.6(2.7)$ & 38.4 & $97.6(3.5)$ & 54.4 & $97.6(0.5)$ \\
\hline & 25.0 & 67.6 & $74.4(0.5)$ & 64.0 & $112(5.7)$ & 74.4 & $89.4(0.5)$ \\
\hline & 200 & 192 & $71.3(2.1)$ & 206 & $84.8(2.1)$ & 205 & $76.5(3.3)$ \\
\hline \multirow[t]{4}{*}{ River water } & 0.00 & 110 & & 20.0 & & 97.0 & \\
\hline & 5.00 & 115 & 105 (3.6) & 25.5 & $109(6.7)$ & 101 & $80.8(6.2)$ \\
\hline & 50.0 & 149 & $78.3(1.9)$ & 71.0 & $102(3.3)$ & 144 & $93.1(4.6)$ \\
\hline & 400 & 474 & $91.1(6.1)$ & 432 & $103(6.7)$ & 537 & $110(6.4)$ \\
\hline \multirow[t]{4}{*}{ Seawater } & 0.00 & 11.0 & & 16.0 & & 11.0 & \\
\hline & 2.50 & 13.8 & $112(6.9)$ & 18.4 & $95.3(5.1)$ & 13.4 & $94.0(2.6)$ \\
\hline & 25.0 & 33.4 & $89.9(5.8)$ & 36.8 & $83.0(9.3)$ & 34.7 & $94.7(3.3)$ \\
\hline & 200 & 207 & $98.2(8.8)$ & 182 & $82.8(7.6)$ & 195 & $92.2(0.9)$ \\
\hline
\end{tabular}


Table S8. Comparison of proposed method with reference methods for the analysis of target analytes in water samples

\begin{tabular}{|c|c|c|c|c|c|}
\hline \multirow{2}{*}{ Compounds } & \multirow{2}{*}{ Methods } & \multicolumn{4}{|c|}{ Detected $(\mu \mathrm{g} / \mathrm{L})$} \\
\hline & & Farmland water & Seawater & Furong lake water & Maolin lake water \\
\hline \multirow[t]{2}{*}{$\mathrm{CLO}$} & MBS/ITMA-HPLC/DAD a & ND & $0.045(6.4)$ & ND & ND \\
\hline & SPE-HPLC/MS-MS b & ND & $0.043(5.6)$ & ND & ND \\
\hline \multirow[t]{2}{*}{ CAR } & MBS/ITMA-HPLC/DAD & ND & ND & ND & ND \\
\hline & SPE-HPLC/MS-MS & ND & ND & ND & ND \\
\hline \multirow[t]{2}{*}{ ISO } & MBS/ITMA-HPLC/DAD & ND & $0.016(7.0)$ & $0.096(9.5)$ & $0.019(7.4)$ \\
\hline & SPE-HPLC/MS-MS & ND & $0.019(8.6)$ & $0.090(2.3)$ & $0.022(3.3)$ \\
\hline \multirow[t]{2}{*}{ FEN } & MBS/ITMA-HPLC/DAD & $1.2(1.8)$ & $0.47(4.4)$ & $0.41(7.6)$ & $0.23(4.4)$ \\
\hline & SPE-HPLC/MS-MS & $1.4(5.2)$ & $0.55(6.5)$ & $0.41(7.1)$ & $0.22(4.0)$ \\
\hline \multirow[t]{2}{*}{ TRN } & MBS/ITMA-HPLC/DAD & $\mathrm{ND}$ & ND & ND & ND \\
\hline & LLME-HPLC/ & ND & ND & ND & ND \\
\hline \multirow[t]{2}{*}{ MYC } & MBS/ITMA-HPLC/DAD & ND & $0.012(9.2)$ & ND & ND \\
\hline & LLME-GC/MS & ND & $0.015(6.9)$ & ND & ND \\
\hline \multirow[t]{2}{*}{ TRF } & MBS/ITMA-HPLC/DAD & ND & ND & ND & ND \\
\hline & DLLME-GC/MS & ND & ND & ND & ND \\
\hline \multirow[t]{2}{*}{ HEX } & MBS/ITMA-HPLC/DAD & ND & ND & ND & ND \\
\hline & DLLME-GC/MS & ND & ND & ND & ND \\
\hline \multirow[t]{2}{*}{$\mathrm{Cd}^{2+}$} & MBS/ITMA-GFAAS a & $0.011(7.2)$ & $0.020(3.9)$ & $0.023(5.8)$ & $0.019(9.2)$ \\
\hline & SPE-FAAS ${ }^{d}$ & $0.012(1.2)$ & $0.023(8.2)$ & $0.019(3.0)$ & $0.020(1.3)$ \\
\hline \multirow[t]{2}{*}{$\mathrm{Pb}^{2+}$} & MBS/ITMA-GFAAS & $0.010(9.1)$ & $0.033(6.5)$ & ND & ND \\
\hline & SPE-FAAS & $0.013(1.2)$ & $0.037(7.2)$ & ND & ND \\
\hline \multirow[t]{2}{*}{$\mathrm{Cu}^{2+}$} & MBS/ITMA-GFAAS & $0.025(8.5)$ & $0.094(2.2)$ & $0.035(3.1)$ & $0.083(1.8)$ \\
\hline & SPE-FAAS & $0.023(3.2)$ & $0.102(6.3)$ & $0.031(2.3)$ & $0.092(5.1)$ \\
\hline
\end{tabular}

a: Current method;

b: Standard determination method for carbamates pesticides in water (HJ827-2017);

c: The detailed determination procedure can be found in the reference (Analytica Chimica Acta 1024 (2018) 1-17).

d: Standard determination method for heavy metal ions in water (GB 7475-87). 


\section{Standards and Reagents}

AMD (99\%) was supplied by Lanzhou Zhongke Kaite Chemical Co. LTD (Lanzhou, China); AT (98\%), DB (80\%) and ED (98\%) were purchased from Alfa Aesar Ltd. (Tianjin, China); Azobisisobutyronitrile (AIBN, 97\%), ethylenediamine tetraacetic acid disodium salt (EDTA, 99.0\%), analytical grade 1-propanol (99.5\%), 1,4-butanediol, formic acid (FA) and guaranteed reagent nitric acid (68\%) were bought from Xilong Chemical Co. (Guangzhou, China); HPLC-grade acetonitrile $(\mathrm{ACN})$ and methanol were purchased from Tedia (Fairfield, USA). Milli-Q purification system (Merck Millipore, Germany) was employed to prepare the ultrapure water used in the present study.

The carbamates pesticides used in the study including cloethocarb (CLO, 99\%) and fenoxycarb (FEN, 99.5\%) were obtained from Dr. Ehrenstorfer (Germany). Isoprocarb (ISO, 98\%) and carbaryl (CAR, 98\%) were bought from Aladdin Industrial Corporation (Shanghai, China); Four triazoles pesticides including triadimenol (TRN, 98\%), myclobutanil (MYC, 98\%), triadimefon (TRF, 99\%) and hexaconazole (HEX, 99\%) were supplied by Haling Biological Technology Co. (Shanghai, China). The stock standard solutions $(1000 \mathrm{mg} / \mathrm{L})$ of each pesticide were prepared by dissolving certain amount of respective compounds in methanol and stored at $4^{\circ} \mathrm{C}$. The working solutions at various concentrations were prepared by step-by-step dilution with methanol. Standard solutions of metal ions were prepared using ultrapure water as solvent by the same as the above-mentioned procedure of pesticides.

\section{Instruments}

The MBS was characterized with Fourier transform infrared spectrum (FT-IR, Avatar-360, Thermo Nicolet, Madison, WI, USA), elemental analysis (EA) (PerkinElmer, Model PE 2400, Shelton, CT, USA), scanning electron microscopy 
(SEM) (Philips, Eindhoven, the Netherlands), and mercury intrusion porosimeter (MIP) Model PoreMaster-60 (Quantachrome Instruments, Florida, USA). The parameters of the MIP are as follows:

The range of pressure, $0-60000 \mathrm{psi}$; the range of pore size: $0.0035-400 \mu \mathrm{m} ;$; resolution ratio, $0.001 \mathrm{cc}$; sample volume, $1-100 \mathrm{cc}$; accuracy, $0.25 \%$.

The specific surface area of MBS was calculated from nitrogen adsorption/desorption measurements of dry bulk monoliths using a BSD-PM2 surface area analyzer (Beishide Instrument Technology Co., Ltd., Beijing, China)

Chromatographic conditions and AAS information for the analysis of studied pesticides and metal ions

Chromatographic analysis of studied pesticides was performed on an HPLC system (Thermo Fisher Scientific, USA) equipped with an LPG-3400SDN quaternary pump and SPD-20A UV/VIS detector. WPS-3000 automatic sample injector with $20 \mu \mathrm{L}$ loop (WPS-300SL) was used to carry out sample injection. A Phenomenex Luna C18 column $(250 \times 4.6 \mathrm{~mm}$ i.d., $5 \mu \mathrm{m}$ particle size $)$ was used for separation of the pesticides. Ultrapure water and $\mathrm{ACN}(45 / 55, \mathrm{v} / \mathrm{v})$ were used as mobile phase components at a flow rate of $1.0 \mathrm{~mL} / \mathrm{min}$.

ICE 300 Series atomic absorption spectrometer (Thermo Fisher Scientific, Germany) with a GF35 graphite furnacen atomizer and AC1200 coolant circulatory (Beijing Allway Co., China), and equipped with deuterium background correction was utilized for the determination of three metal ions. The instrumental conditions for the analysis are listed in Supporting Information (Table S2).

\section{The measurement of the adsorption capacity of MBS}

$\mathrm{Cu}$ (II) and CAR were selected as test target analytes to evaluate the adsorption capacity of MBS for heavy metal ions and pesticides, respectively. In the experiment, 
the initial concentrations of $\mathrm{Cu}$ (II) and CAR were 20 and $500 \mu \mathrm{g} / \mathrm{L}$, respectively, and the water sample was $50 \mathrm{~mL}$. During enrichment of the two target compounds by MBS, the solution after treatment of MBS was collected in the beakers. The residual concentrations of $\mathrm{Cu}$ (II) and CAR in the solution were measured by GFAAS and HPLC/DAD, respectively.

The adsorption capacity Q $(\mu \mathrm{g} / \mathrm{g})$ of MBA was calculated as follows:

$$
\mathrm{Q}=\left(\mathrm{C}_{0}-\mathrm{C}_{1}\right) \times \mathrm{V} / \mathrm{m}
$$

where $\mathrm{C}_{0}(\mu \mathrm{g} / \mathrm{L})$ and $\mathrm{C}_{1}(\mu \mathrm{g} / \mathrm{L})$ were the initial and residual concentrations of the target analytes, respectively. $\mathrm{V}(\mathrm{mL})$ and $\mathrm{m}(\mathrm{mg})$ were the volume of the solution $(50$ $\mathrm{mL}$ in this work) and the mass of MBS (4 mg), respectively. According to the equation, the adsorption capacities of MBS for $\mathrm{Cu}$ (II) and CAR were 164 and 6022 $\mu \mathrm{g} / \mathrm{g}$, respectively.

\section{References:}

(1) Chen, L,; Li, M.; Ai, Y. H.; Dang, X. P.; Huang, J. L.; Chen, H. X. Food Chem. 2018, 269, 181-186.

(2) Sun, B.; Wang, C. P.; Wang, Q.; Chen, L.; Dang, X. P.; Huang, J. L. Chen, H. X. Food Anal. Methods 2017, 10, 3847-3855.

(3) Arnnok, P.; Patdhanagul, N.; Burakham, R. Chromatographia 2015, 78, 1327-1337.

(4) Pourreza, N.; Hoveizavi, R. Anal. Chim. Acta 2005, 549, 124-128.

(5) Zheng, F.; Hu, B. Anal. Chim. Acta 2007, 605, 1-10.

(6) Yamini, Y.; Hassan, J.; Karbasi, M. H. Microchim. Acta 2004, 148, 305-309.

(7) Chen, L.; Pei, J. X.; Huang, X. J.; Lu, M. J. Chromatogr. A 2018, 1564, 34-41.

(8) Lin, S. L.; Wu, Y. R.; Fuh, MPo. R. Talanta 2016, 147, 199-206.

(9) Jin, T. T.; Li, F.; Cheng, J.; Wu, S. J.; Zhou, H. B.; Cheng, M. Microchim. Acta 2016, 183, 543-551.

(10) Carrera, S.; Santiago, G.; Vega, M. Microchem. J. 2016, 129, 133-136.

(11) Wang, M. Y.; Yan, H. Y.; Yuan, Y. N.; Han, Y. H. Anal. Bioanal. Chem. 2015, 407, 1231-1239.

(12) Zhao, L. Y.; Fei, J. J.; Lian, H. Z.; Mao, L.; Cui, X. B. J. Anal. At. Spectrom. 2019, 34, 1693-1700.

(13) Wang, C.; He, M.; Chen, B. B.; Hu, B. Talanta 2018, 188, 736-743.

(14) Yao, W. X.; Fan, Z. F.; Zhang, S. L. J. Sep. Sci. 2019, 42, 2679-2686.

(15) Pang, J. L.; Mei, M.; Yuan, D. X.; Huang, X. J. Talanta 2018, 184, 411-417. 